Research Article

\title{
Identification of Rational Systems with Logarithmic Quantized Data
}

\author{
Mingming Ji $(D)$ and Shengchao Su \\ Laboratory of Intelligent Control and Robotics, Shanghai University of Engineering Science, Shanghai 201620, China \\ Correspondence should be addressed to Mingming Ji; jimingming923@163.com
}

Received 22 March 2021; Revised 17 May 2021; Accepted 24 June 2021; Published 13 July 2021

Academic Editor: Frederic Kratz

Copyright (C) 2021 Mingming Ji and Shengchao Su. This is an open access article distributed under the Creative Commons Attribution License, which permits unrestricted use, distribution, and reproduction in any medium, provided the original work is properly cited.

\begin{abstract}
This paper is concerned with the quantized identification of rational systems, where the systems' output is quantized by a logarithmic quantizer. Under the assumption that the systems' input is periodic, the identification procedure is categorized into two steps. The first step is to identify the noise-free output of systems based on the quantized data. The second is to identify the unknown parameter based on the input and the estimation of the noise-free output. The identification algorithm is also summarized. Asymptotic convergence of the estimators is analyzed in detail, which shows that the estimators are convergent almost everywhere. A numerical example is given to illustrate the results obtained in this paper.
\end{abstract}

\section{Introduction}

Due to sensor limitations, signal quantization, or coding for the limitation of networked bandwidth in modern control systems, the study of system identification of plants with quantized observations becomes significant in modeling systems. The quantization may affect the algorithm of system identification, the convergence of the estimates, and so on. Thus, more attention should be paid when there exist quantized data in a system.

In recent years, the research of system identification with quantized data has constituted a vast body of research. For example, Wang et al. [1] presented some new methodologies for the identification of a system with quantized output observations and explored their potential in extending control capabilities for systems with limited information or networked systems. Gustafsson and Karlsson [2] studied fundamental issues in estimation that related directly to the core methods of identification for the system based on quantized observations. The identification of ARMA models was studied in [3], where the systems' outputs were subject to both quantization and packet dropout. Maximum likelihood estimation was analyzed in [4], where dithering noise was added before quantization. Widrow and Kollar $[5,6]$ developed the statistic theory of uniform quantized effect. Identification of the system with only binary-valued sensors was investigated in [7]. Based on the Gauss-Markov theorem, a quantile-based estimator was proposed in [8]. More research about system identification using quantized data can be seen in [9-15].

It has to be pointed out that most of the above literatures concerning with quantized identification were based on the uniform quantized data. Besides the uniform quantizer, there is logarithmic quantizer [16-19], dynamic quantizer [20-24], and so on. Compared with uniform quantizer, sometimes these quantizers have better quantization effect. However, the related research literature is quite limited to the study of system identification with other types of quantized data. Since logarithmic quantizer has the advantage that it has the better efficiency on data rate for performance control, it has been widely studied and used in control theory. Thus, it is necessary to study the identification of systems based on logarithmic quantized data. In this paper, we will analyze the rational system identification concerning that the output data are quantized by a logarithmic quantizer.

For the identification of rational systems with quantized data, in [1], a two-step identification procedure was 
introduced that employs periodic signals, empirical measures, and identification features so that rational models can be identified without resorting to complicated nonlinear search algorithms. The present work of this paper extends that of [1] in the following three ways. (1) The identification of rational systems is studied based on the logarithmic quantized data. This quantized strategy is more complex compared to [1], where the systems' output was observed via a binary-valued sensor. (2) The difficulty in treating logarithmic quantized data is that the logarithmic quantization levels are not uniform. Then, the method of quantile-based estimation is used to identify the noise-free output of system. (3) A fundamental problem of system identification with quantized data is that the convergence should be satisfied. Then, the convergence almost everywhere of the estimators for both the noise-free output and the unknown parameter vector of the system are analyzed in detail.

This paper begins with a statement of the problem in Section 2, where the descriptions of the rational systems and the logarithmic quantizer are given, respectively. How to identify the unknown parameters is analyzed in Section 3. The convergence of the parameter estimates is established in
Section 4. In Section 5, some simulation results are presented. Concluding remarks are given in Section 6.

Notations. Throughout this paper, the superscript " $T$ " denotes the transpose of a vector or a matrix. $|\cdot|$ stands for Euclidean norm. $\|\cdot\|$ represents the largest singular value of the corresponding matrix. $\operatorname{Pr}(\cdot)$ and $E(\cdot)$ represent the probability and mathematical expectation of the corresponding random variables, respectively. $Z$ denotes the set of integers. i.i.d. denotes independent identically distributed.

\section{Problem Statement}

In this paper, consider the following system which is disturbed by noise:

$$
y_{k}=x_{k}+d_{k}, \quad k=1,2, \ldots,
$$

where $x_{k}=G(q) u_{k}, q$ is the one-step backward shift operator satisfying $q u_{k}=u_{k-1}, d_{k}$ is the random sensor noise, and $G(q)$ is a function of $q$ described by

$$
G(q)=\frac{B(q)}{1-A(q)}=\frac{b_{1} q+b_{2} q^{2}+\cdots+b_{m} q^{m}}{1-\left(a_{1} q+a_{2} q^{2}+\cdots+a_{m} q^{m}\right)}, \quad m=1,2, \ldots, k
$$

System (1) can also be expressed in its regression form:

$$
y_{k}=A(q) y_{k}+B(q) u_{k}+(1-A(q)) d_{k}=\Phi_{k}^{T} \theta+\tilde{d}_{k},
$$

where

$$
\begin{aligned}
\Phi_{k} & =\left[y_{k-1}, y_{k-2}, \ldots, y_{k-m}, u_{k-1}, u_{k-2}, \ldots, u_{k-m}\right]^{T}, \\
\theta & =\left[a_{1}, a_{2}, \ldots, a_{m}, b_{1}, b_{2}, \ldots, b_{m}\right]^{T}, \\
\tilde{d}_{k} & =(1-A(q)) d_{k},
\end{aligned}
$$

where $\theta$ is the unknown parameter vector which needs to be identified.

Remark 1. Compared with equation disturbance structure,

$$
y_{k}+a_{1} y_{k-1}+a_{2} y_{k-2}+\cdots+a_{m} y_{k-m}=b_{1} u_{k-1}+b_{2} u_{k-2}+\cdots+b_{m} u_{k-m}+d_{k} \text {, }
$$

which is an autoregressive moving average (ARMA) model structure, the structure of system (1) can relate the systems' output to sensor measurement errors in practical system configurations [1]. Thus, we adopt system (1) which is the structure of output disturbance in this paper.

Due to the limitation of the network bandwidth, suppose that the output $y_{k}$ of the system is measured by a logarithmic quantizer, i.e.,

$$
s_{k}=Q\left(y_{k}\right)
$$

where $Q(\cdot)$ is a logarithmic quantizer with quantization density $\rho \in(0,1)$. In other words, the actual output of the system is $s_{k}$. The structure of the system studied in this paper is illustrated in Figure 1.

The quantizer $Q(\cdot)$ has the following quantization levels:

$$
U=\left\{ \pm \rho^{i} v_{0}, i=0, \pm 1, \pm 2, \ldots\right\} \cup\{0\},
$$

where $v_{0}>0$ is a scaling parameter. $Q(\cdot)$ can be expressed in the following form: 


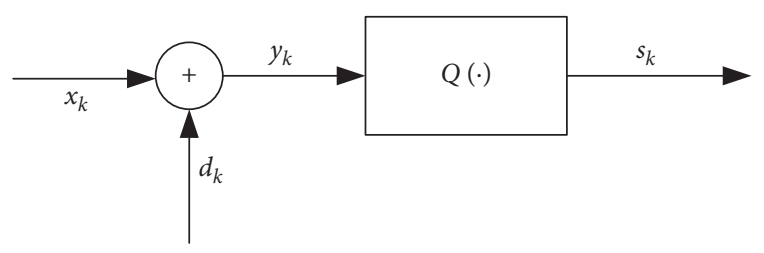

FIGURE 1: The structure of the system.

$$
Q(\varepsilon)= \begin{cases}\rho^{i} v_{0}, & \varepsilon \in\left[\frac{\rho+1}{2} \rho^{i-1} v_{0}, \frac{\rho+1}{2} \rho^{i} v_{0}\right), \\ -\rho^{i} v_{0}, & \varepsilon \in\left(-\frac{\rho+1}{2} \rho^{i} v_{0},-\frac{\rho+1}{2} \rho^{i-1} v_{0}\right], \\ 0, & \varepsilon=0,\end{cases}
$$

where $i \in Z$. This type of quantizer is depicted in Figure 2 .

The purpose of this paper is to identify the unknown parameter vector $\theta$ from the logarithmic quantized output data $s_{k}(k=1,2, \ldots$,$) and then establish the corresponding$ convergence properties. The identification procedure will be categorized into two steps. The first step is to identify the noise-free output $x_{k}$ based on the logarithmic quantized output data $s_{k}$. The second is to identify the unknown parameter vector $\theta$ on the basis of the input $u_{k}$ and the estimation of $x_{k}$.

\section{Parameter Identification}

For system (1), suppose that the input $u_{k}$ is $2 m$-periodic. Since $G(q)$ is a stable function. Then, after a short transient duration, the noise-free output $x_{k}=G(q) u_{k}$ of system (1) will also be $2 m$-periodic, i.e., for any nonnegative integer $l=0,1, \ldots$ and positive integers $j=1,2, \ldots, 2 m$, $x_{j+2 m l}=x_{j}$. Since $x_{k}$ is $2 m$-periodic, we can suppose that the first $2 m$ unknown real numbers of $x_{k}$ are $\gamma^{\{j\}}$, i.e., $x_{j}=\gamma^{\{j\}}$. Then, the identification of $x_{k}$ is converted into the identification of the $2 m$ real numbers $\gamma^{\{j\}}$.

Remark 2. It should be mentioned that the number of parameters to be identified is $2 \mathrm{~m}$. And, we also select the input to be $2 m$-periodic. If the input is not $2 m$-periodic, then the method proposed in this paper is not feasible.

Based on the above assumption and analysis, for $j=1,2, \ldots, 2 m$, the output of the system can be expressed as follows:

$$
y_{j+2 l m}=x_{j+2 l m}+d_{j+2 l m}=\gamma^{\{j\}}+d_{j+2 l m}, \quad l=0,1, \ldots
$$

Then, the quantized output $s_{k}$ is

$$
s_{j+2 l m}=Q\left(y_{j+2 l m}\right)=Q\left(\gamma^{\{j\}}+d_{j+2 l m}\right), \quad l=0,1, \ldots
$$

Now, the identification procedure is converted into identifying $\gamma^{\{j\}}$ based on $s_{k}$, firstly, and then identifying $\theta$ based on the estimate of $\gamma^{\{j\}}$ and the input $u_{k}$.

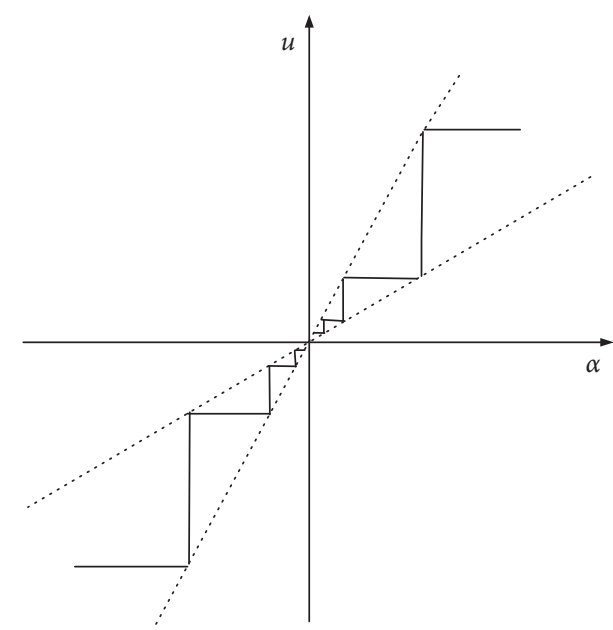

FIGURE 2: Logarithmic quantizer.

3.1. Identification of $x_{k}$. The analysis of the identification of $x_{k}$ is based on the following assumption.

Assumption 1. For the random sensor noise $d_{k}(k=1,2, \ldots$,$) proposed in system (1),\left\{d_{k}\right\}$ is a sequence of i.i.d. random variables. Moreover, the distribution function $F(\cdot)$ of $d_{k}$ is invertible and its inverse function $F^{-1}(\cdot)$ is twice continuously differentiable.

For system (10), the quantized output $s_{k}$ can be described as follows:

$$
s_{j+2 l m}= \begin{cases}\rho^{i} v_{0}, & y_{j+2 l m} \in\left[\frac{\rho+1}{2} \rho^{i-1} v_{0}, \frac{\rho+1}{2} \rho^{i} v_{0}\right), \\ -\rho^{i} v_{0}, & y_{j+2 l m} \in\left(-\frac{\rho+1}{2} \rho^{i} v_{0},-\frac{\rho+1}{2} \rho^{i-1} v_{0}\right], \\ 0, & y_{j+2 l m}=0 .\end{cases}
$$

Remark 3. According to expression (11), the possible values of $s_{k}$ are $\pm \rho^{i} v_{0}, 0$ with $i \in Z$. Since $\lim \left( \pm \rho^{i} v_{0}\right)=0$ and $\operatorname{Pr}\left\{s_{j+2 l m}=0\right\}=0$, then, in this paper, we will only analyze the probability of the event $s_{j+2 i m} \leq \pm \rho^{j} v_{0}$.

In this paper, the method of quantile-based estimation which is proposed in [8] is used to estimate $\gamma^{\{j\}}$.

For $j=1,2, \ldots, 2 m$, denote

$$
p^{\{j\}}= \begin{cases}\operatorname{Pr}\left\{s_{j+2 l m} \leq \rho^{i} v_{0}\right\}, & \text { if } s_{j+2 l m} \geq 0, \\ \operatorname{Pr}\left\{s_{j+2 l m} \leq-\rho^{i} v_{0}\right\}, & \text { if } s_{j+2 l m}<0 .\end{cases}
$$

When $s_{j+2 l m} \geq 0$, according to expression (11),

$$
p^{\{j\}}=\operatorname{Pr}\left\{s_{j+2 l m} \leq \rho^{i} v_{0}\right\}=\operatorname{Pr}\left\{y_{j+2 l m}<\frac{\rho+1}{2} \rho^{i} v_{0}\right\} .
$$
then

Since $y_{k}$ can be expressed as $y_{j+2 l m}=\gamma^{\{j\}}+d_{j+2 l m}$, 


$$
p^{\{j\}}=\operatorname{Pr}\left\{\gamma^{\{j\}}+d_{j+2 l m}<\frac{\rho+1}{2} \rho^{i} v_{0}\right\}=\operatorname{Pr}\left\{d_{j+2 l m}<\frac{\rho+1}{2} \rho^{i} v_{0}-\gamma^{\{j\}}\right\} .
$$

Thus,

$$
p^{\{j\}}=F\left(\frac{\rho+1}{2} \rho^{i} v_{0}-\gamma^{\{j\}}\right)
$$

where $F(\cdot)$ is the distribution of the random sensor noise $d_{k}$. In the similar way, when $s_{j+2 l m}<0$,

$$
p^{\{j\}}=F\left(-\frac{\rho+1}{2} \rho^{i-1} v_{0}-\gamma^{\{j\}}\right) .
$$

Denote $C_{i}=\left\{\begin{array}{ll}\rho^{i} v_{0} & \text { if } s_{j+2 l m} \geq 0 \\ -\rho^{i} v_{0} & \text { if } s_{j+2 l m}<0\end{array}, i \in Z\right.$, and define the integers $a_{i}$ and $b_{i}$ as follows:

$$
\begin{aligned}
& a_{i}= \begin{cases}1, & \text { if } C_{i}>0, \\
-1, & \text { if } C_{i}<0,\end{cases} \\
& b_{i}= \begin{cases}0, & \text { if } C_{i}>0, \\
1, & \text { if } C_{i}<0 .\end{cases}
\end{aligned}
$$

Then, the probability $p^{\{j\}}$ can be expressed as

$$
p^{\{j\}}=\operatorname{Pr}\left\{s_{j+2 l m} \leq C_{i}\right\}=F\left(\frac{\rho+1}{2 \rho^{b_{i}}} a_{i} C_{i}-\gamma^{\{j\}}\right) .
$$

For some positive integer $N$, let the observation length be $2 m N$. Given $j=1,2, \ldots, 2 m$, define

$$
\widehat{p}_{N}^{\{j\}}=\frac{1}{N} \sum_{l=0}^{N-1} I_{\left\{s_{j+2 l m} \leq C_{i}\right\}}
$$

where $I_{\{\cdot\}}$ is the indicator function. It is described as

$$
I_{\left\{s_{k} \in A\right\}}= \begin{cases}1, & \text { if } s_{k} \in A, \\ 0, & \text { otherwise. }\end{cases}
$$

Letting $p^{\{j\}}=\widehat{p}_{N}^{\{j\}}$, the estimator $\widehat{\gamma}_{N}^{\{j\}}$ of the parameter $\gamma^{\{j\}}$ can be obtained as follows:

$$
\widehat{\gamma}_{N}^{\{j\}}=\frac{\rho+1}{2 \rho^{b_{i}}} a_{i} C_{i}-F^{-1}\left(\widehat{p}_{N}^{\{j\}}\right) .
$$

Then, the estimator of $x_{k}$ is obtained, i.e.,

$$
\widehat{x}_{j}=\widehat{x}_{j+2 l m}=\widehat{\gamma}_{N}^{\{j\}}=\frac{\rho+1}{2 \rho^{b_{i}}} a_{i} C_{i}-F^{-1}\left(\widehat{p}_{N}^{\{j\}}\right) .
$$

3.2. Identification of $\theta$. The analysis of the identification of $\theta$ is based on the following assumption.

Assumption 2. For $A(q)$ and $B(q)$ in system (1), $1-A(q)$ and $B(q)$ are coprime polynomials.

Remark 4. It should be mentioned that Assumption 1 and Assumption 1 do not give too many restrictions on system (1). Thus, the method proposed in this paper is feasible for most systems.
System (1) without noise is described as

$$
y_{k}=x_{k}=\Phi_{k}^{T} \theta
$$

Since the system has a $2 m$-periodic input, for some $k_{0}=$ $1,2, \ldots$, we can define

$$
\begin{aligned}
& X=\left[x_{k_{0}}, x_{k_{0}+1}, \ldots, x_{k_{0}+2 m-1}\right]^{T}, \\
& \Pi=\left[\Phi_{k_{0}}, \Phi_{k_{0}+1}, \ldots, \Phi_{k_{0}+2 m-1}\right]^{T} .
\end{aligned}
$$

Then, system (1) without noise can be denoted as

$$
X=\Pi \theta .
$$

For this system, if $\Pi$ is full rank, then there exists a oneto-one mapping between the output $X$ and the parameter vector $\theta$. The property of the matrix $\Pi$ is illustrated in the following lemma.

Lemma 1 (see [1]). Under Assumption 2, if the system's input $u_{k}$ is $2 m$-periodic and full rank, then $\Pi$ is invertible for all $k_{0}$ and $\left\|\Pi^{-1}\right\|$ is independent of $k_{0}$.

To estimate $\theta$, we can define

$$
\begin{aligned}
\widehat{X} & =\left[\widehat{x}_{k}, \widehat{x}_{k+1}, \ldots, \widehat{x}_{k+2 m-1}\right]^{T}, \\
\widehat{\Phi}_{k} & =\left[\widehat{x}_{k-1}, \widehat{x}_{k-2}, \ldots, \widehat{x}_{k-m}, u_{k-1}, u_{k-2}, \ldots, u_{k-m}\right]^{T}, \\
\widehat{\Pi} & =\left[\widehat{\Phi}_{k}, \widehat{\Phi}_{k+1}, \ldots, \widehat{\Phi}_{k+2 m-1}\right]^{T} .
\end{aligned}
$$

Let

$$
\widehat{x}_{k}=\widehat{\Phi}_{k}^{T} \widehat{\theta}
$$

where $\hat{\theta}$ is the estimator of $\theta$. Then, the following expression is obtained:

$$
\widehat{X}=\widehat{\Pi} \widehat{\theta}
$$

Thus, the least-square estimator of $\theta$ is

$$
\widehat{\theta}=\left(\widehat{\Pi}^{T} \widehat{\Pi}\right)^{-1} \widehat{\Pi}^{T} \widehat{X}
$$

3.3. Summary of the Identification Algorithm. The identification steps of the parameter $\theta$ are summarized Algorithm 1 .

\section{Asymptotic Analysis}

In this section, we will analyze the statistical properties of the estimators $\widehat{x}_{k}$ and $\hat{\theta}$ when the sample number $N$ tends to infinity, i.e., the convergence of the estimators $\widehat{x}_{k}$ and $\widehat{\theta}$. To proceed, some definitions and lemmas are presented.

Definition 1 (convergence almost everywhere, see [25]). A sequence $\left\{\xi_{n}(\omega)\right\}$ is said to converge almost everywhere to $\xi(\omega)$ if $\operatorname{Pr}\left\{\lim _{n \longrightarrow \infty} \xi_{n}(\omega)=\xi(\omega)\right\}=1$. Denote it as $\xi_{n}(\omega) \longrightarrow^{a} . s . \xi(\omega)$. 
Lemma 2 (Kolmogorov strong law of large numbers, see [25]). Let $\left\{\xi_{i}\right\}(i=1,2, \ldots$,$) be an i.i.d. sequence of random$ variables; then $(1 / n) \sum_{i=1}^{n} \xi_{i} \longrightarrow^{a}$.s. $\mu$ if and only if $E\left[\xi_{i}\right]=\mu<\infty$.

4.1. Asymptotic Analysis of $\hat{x}_{k}$. The convergence of the estimator will be illustrated in the following theorem.

Theorem 1. Under Assumption 1, the estimator obtained in (23) satisfies that

$$
\widehat{x}_{j+2 l m}=\widehat{\gamma}_{N}^{\{j\} \stackrel{a}{\longrightarrow} . s .} \gamma^{\{j\}}, \quad j=1,2, \ldots, m .
$$

Proof. According to the definition of the indicator function, it is easily obtained that $I_{\left\{s_{j+2 l m} \leq C_{i}\right\}}(j=1,2, \ldots, m)$ are Bernoulli random variables. Moreover,

$$
E\left\{I_{\left\{s_{j+2 l m} \leq C_{i}\right\}}\right\}=1 \times \operatorname{Pr}\left\{s_{j+2 l m} \leq C_{i}\right\}+0 \times \operatorname{Pr}\left\{s_{j+2 l m}>C_{i}\right\}=p^{\{j\}} .
$$

It is obvious that

$$
E\left\{\widehat{p}_{N}^{\{j\}}\right\}=p^{\{j\}}
$$

According to the Kolmogorov strong law of large numbers, it follows that

$$
\widehat{p}_{N}^{\{j\}} \stackrel{\text { a.s. }}{\longrightarrow} p^{\{j\}} .
$$

Furthermore, Assumption 1 implies that $F^{-1}(\cdot)$ is continuous. Then,

$$
F^{-1}\left(\widehat{p}_{N}^{\{j\}}\right) \stackrel{\text { a.s. }}{\longrightarrow} F^{-1}\left(p^{\{j\}}\right)
$$

i.e.,

$$
F^{-1}\left(\widehat{p}_{N}^{\{j\}}\right) \stackrel{\text { a.s. }}{\longrightarrow} \frac{\rho+1}{2 \rho^{b_{i}}} a_{i} C_{i}-\gamma^{\{j\}} .
$$

Thus,

$$
\frac{\rho+1}{2 \rho^{b_{i}}} a_{i} C_{i}-F^{-1}\left(\widehat{p}_{N}^{\{j\}}\right) \stackrel{\text { a.s. }}{\longrightarrow} \gamma^{\{j\}},
$$

i.e.,

$$
\widehat{x}_{j+2 l m}=\widehat{\gamma}_{N}^{\{j\}} \stackrel{\text { a.s. }}{\longrightarrow} \gamma^{\{j\}} .
$$

The proof is completed.

4.2. Asymptotic Analysis of $\hat{\theta}$. The convergence of the estimator $\widehat{\theta}$ will be established in the following theorem.

Theorem 2. If Assumption 1 and Assumption 2 hold, then

$$
\widehat{\theta} \stackrel{\text { a.s. }}{\longrightarrow} \theta \text {. }
$$

proof. $\widehat{X}=\widehat{\Pi} \hat{\theta}$ can be expressed as

$$
X+E_{N}=\left(\Pi+f\left(E_{N}\right)\right) \widehat{\theta},
$$

where $E_{N}$ and $f\left(E_{N}\right)$ are quantization errors, i.e.,

$$
\begin{aligned}
E_{N} & =\widehat{X}-X, \\
f\left(E_{N}\right) & =\widehat{\Pi}-\Pi .
\end{aligned}
$$

According to Theorem 1, it is easily obtained that $E_{N} \longrightarrow{ }^{a}$.s. 0. Then, (30) implies that $f\left(E_{N}\right) \longrightarrow 0$ as $E_{N} \longrightarrow 0$. Thus, $f\left(E_{N}\right) \longrightarrow{ }^{a}$.s. 0. If (30) is left multiplied by the matrix $\Pi^{T}$, then

$$
\Pi^{T} X+\Pi^{T} E_{N}=\left(\Pi^{T} \Pi+\Pi^{T} f\left(E_{N}\right)\right) \widehat{\theta} .
$$

Thus,

$$
\widehat{\theta}=\left(\Pi^{T} \Pi+\Pi^{T} f\left(E_{N}\right)\right)^{-1}\left(\Pi^{T} X+\Pi^{T} E_{N}\right) \stackrel{\text { a.s. }}{\longrightarrow} \theta .
$$

The proof is completed.

\section{Simulations}

In this section, a numerical example is given to illustrate the results obtained in this paper.

Consider the following system:

$$
\begin{aligned}
& y_{k}=x_{k}+d_{k}, \\
& x_{k}=\frac{0.7 q+0.6 q^{2}}{1-0.5 q+0.4 q^{2}} u(k),
\end{aligned}
$$

where the disturbance $d_{k}(k=1,2, \ldots$,$) is uniformly dis-$ tributed in the interval $[-0.1,0.1]$.

Then, the regression model of this system is obtained as

$$
y_{k}=\Phi_{k}^{T} \theta+\tilde{d}_{k}
$$

where $\Phi_{k}=\left[\begin{array}{llll}y_{k-1} & y_{k_{-} 2} & u_{k-1} & u_{k-2}\end{array}\right]^{T}$, the parameter $\theta=\left[\begin{array}{llll}0.5 & -0.4 & 0.7 & 0.6\end{array}\right]^{T^{2}}$, and the noise $\widetilde{d}_{k}=d_{k}-$ $0.5 d_{k-1}+0.4 d_{k-2}$. Suppose that the output $y_{k}$ is quantized by the logarithmic quantizer $Q(\cdot)$ which is described as (8) with the quantization density $\rho=0.95$ and the scaling parameter $v_{0}=1$. Then, $s_{k}=Q\left(y_{k}\right)$.

For a 4-periodic input $u_{k}$, using Matlab to simulate this system, it is obtained that $x_{k}=G(q) u_{k}$ is also 4-periodic after a short transient duration. Moreover, generate a group of $d_{k}$; then, a group of $s_{k}$ is obtained. According to Algorithm 1, the estimated errors of the parameters are illustrated in Figures 3 and 4, respectively, which show that the errors converge to 0 quickly. Thus, the results obtained in this paper are effective. 
Step 1: if $s_{j+l m} \geq 0$, let $C_{i}=\rho^{i} v_{0}$, if $s_{j+l m}<0$, let $C_{i}=-\rho^{i} v_{0}$.

Step 2: calculate $a_{i}$ and $b_{i}$ using formulas (17) and (18), respectively.

Step 3: calculate $\widehat{p}_{N}^{\{j\}}$ using formula (19).

Step 4: calculate $\hat{x}_{j+2 l m}$ using formula (21).

Step 5. calculate the estimator using formula (30).

Algorithm 1: Summary of the identification steps.

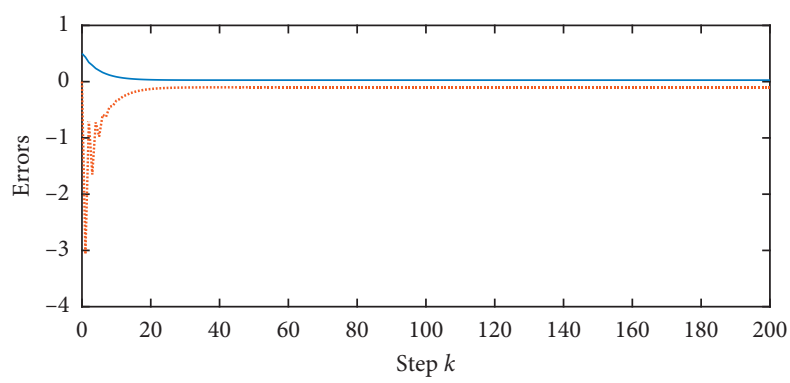

$-a 1$

Figure 3: Estimated errors of $a_{1}=0.5$ and $a_{2}=-0.4$.

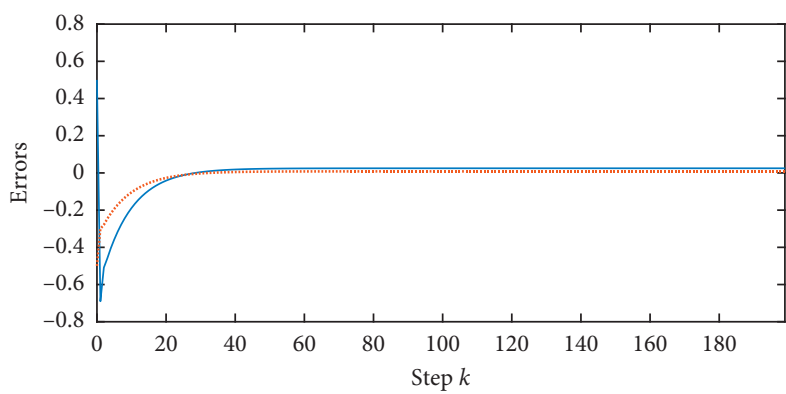

$-b 1$

Figure 4: Estimated errors of $b_{1}=0.7$ and $b_{2}=0.6$.

\section{Conclusion}

This paper has addressed the quantized identification of rational systems, where the systems' output is quantized by a logarithmic quantizer. Assuming that the input is periodic, the identification procedure has been categorized into two steps. For the first step, the quantile-based estimation has been used to estimate the noise-free output of the system. For the second step, the parameter vector has been obtained according to the one-to-one mapping between the noise-free output and the unknown parameter vector. The identification algorithm has also been summarized. In the end, the asymptotic convergence of both the noise-free output and the unknown parameter has been analyzed in detail. Numerical examples have also been given to illustrate the results obtained in this paper.

\section{Data Availability}

No underlying data are used in this paper.

\section{Conflicts of Interest}

The authors declare that they have no conflicts of interest.

\section{Acknowledgments}

This paper was supported by the National Natural Science Foundation of China, under Grant 61603241.

\section{References}

[1] L. Y. Wang, G. G. Yin, J. F. Zhang, and Y. L. Zhao, System Identification with Quantized Observations, Birkhauser, Basel, Switzerland, 2010.

[2] F. Gustafsson and R. Karlsson, "Statistical results for system identification based on quantized observations," Automatica, vol. 45, no. 12, pp. 2794-2801, 2009.

[3] D. Marelli, K. You, and M. Fu, "Identification of ARMA models using intermittent and quantized output observations," Automatica, vol. 49, no. 2, pp. 360-369, 2013. 
[4] F. Gustafsson and R. Karlsson, "Generating dithering noise for maximum likelihood estimation from quantized data," Automatica, vol. 49, no. 2, pp. 554-560, 2013.

[5] B. Widrow, "A study of rough amplitude quantization by means of Nyquist sampling theory," IRE Transactions on Circuit Theory, vol. 3, no. 4, pp. 266-276, 1956.

[6] B. Widrow, I. Kollar, and M.-C. Liu, "Statistical theory of quantization," IEEE Transactions on Instrumentation and Measurement, vol. 45, no. 2, pp. 353-361, 1996.

[7] L. Y. Wang, J. F. Zhang, and G. G. Yin, "System identification using binary sensors," IEEE Transactions on Automatic Control, vol. 48, no. 11, pp. 1892-1907, 2003.

[8] A. Moschitta, J. Schoukens, and P. Carbone, "Parametric system identification using quantized data," IEEE Transactions on Instrumentation and Measurement, vol. 64, no. 8, pp. 2312-2322, 2015.

[9] M. M. Ji and G. Y. Fu, "Identification of systems with logarithmic quantizer," in Proceedings of the 37th Chinese Control Conference, Wuhan, China, July 2018.

[10] P. Carbone, J. Schoukens, and A. Moschitta, "Dynamic signal measurements based on quantized data," IEEE Transactions on Instrumentation and Measurement, vol. 66, no. 2, pp. 223-233, 2017.

[11] J. Guo and J. D. Diao, "FIR system identification under eitheror communication with quantised inputs and quantised output observations," IET Control Theory \& Applications, vol. 13, no. 8, pp. 1204-1210, 2019.

[12] Q. He, G. G. Yin, and L. Y. Wang, "System identification under regular, binary, and quantized observations: moderate deviations error bounds," IEEE Transactions on Automatic Control, vol. 60, no. 6, pp. 1635-1640, 2015.

[13] J. Guo, L. Y. Wang, G. Yin, Y. Zhao, and J.-F. Zhang, "Asymptotically efficient identification of FIR systems with quantized observations and general quantized inputs," Automatica, vol. 57, pp. 113-122, 2015.

[14] R. S. Risuleo, G. Bottegal, and H. Hjalmarsson, "Identification of linear models from quantized data: a midpoint-projection approach," IEEE Transactions on Automatic Control, vol. 65, no. 7, pp. 2801-2813, 2020.

[15] J. Wang and Q. Zhang, "Identification of FIR systems based on quantized output measurements: a quadratic programming-based method," IEEE Transactions on Automatic Control, vol. 60, no. 5, pp. 1439-1444, 2015.

[16] N. Elia and S. K. Mitter, "Stabilization of linear systems with limited information," IEEE Transactions on Automatic Control, vol. 46, no. 9, pp. 1384-1400, 2001.

[17] M. Y. Lihua Xie and L. H. Xie, "The sector bound approach to quantized feedback control," IEEE Transactions on Automatic Control, vol. 50, no. 11, pp. 1698-1711, 2005.

[18] M. Ji, Z. Li, and W. Zhang, "Quantized feedback stabilization of discrete-time linear system with Markovian jump packet losses," Neurocomputing, vol. 158, pp. 307-314, 2015.

[19] Y. Jiang and J. Zhai, "Global sampled-data output feedback stabilization for a class of switched stochastic nonlinear systems with quantized input and unknown output gain," Transactions of the Institute of Measurement and Control, vol. 41, no. 16, pp. 4511-4520, 2019.

[20] R. W. Brockett and D. Liberzon, "Quantized feedback stabilization of linear systems," IEEE Transactions on Automatic Control, vol. 45, no. 7, pp. 1279-1289, 2000.

[21] Y. Zhang, Z. Wang, and F. E. Alsaadi, "Detection of intermittent faults for nonuniformly sampled multi-rate systems with dynamic quantisation and missing measurements,"
International Journal of Control, vol. 93, no. 4, pp. 898-909, 2020.

[22] C. Li and J. Lian, "Stabilization of switched nonlinear systems with dynamic output quantization and disturbances," International Journal of Robust and Nonlinear Control, vol. 30, no. 4, pp. 1679-1695, 2020.

[23] Q. Q. Liu and F. Jin, "LQG control of networked control systems with limited information," Mathematical Problems in Engineering, vol. 2014, Article ID 206391, 12 pages, 2014.

[24] M. L. Corradini and G. Orlando, "Robust quantized feedback stabilization of linear systems," Automatica, vol. 44, no. 9, pp. 2458-2462, 2008.

[25] M. Lo'eve, Probability Theory, Springer-Verlag, Berlin, Germany, 1977. 